\title{
Identification of potential key genes affecting soybean growth under salt stress via transcriptome study
}

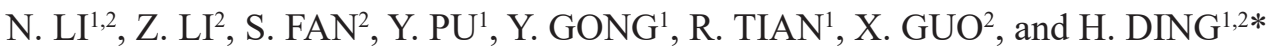 \\ Scientific Research Office, Shandong Center of Crop Germplasm Resources, 250100, Jinan, \\ Shandong, P.R. China ${ }^{1}$ \\ College of Life Science, Shandong Normal University, 250014, Jinan, Shandong, P.R. China ${ }^{2}$
}

\begin{abstract}
Soybean is one of the most important economic crops in the world. However, the salinization of soil results in the decrease of soybean yield as it is only a moderately salt-tolerant crop. We treated three soybean cultivars with low and high concentrations of $\mathrm{NaCl}$. The differentially expressed genes between the control group and the salt treatment group were identified by mRNA sequencing and analyzed by gene ontology and Kyoto encyclopedia of genes and genomes annotations. We performed weighted gene co-expression network analysis on all samples and found genes most related to the phenotype. After verifying the results of differentially expressed genes by quantitative PCR, we finally identified Glyma06G01990, Glyma08G22730, Glyma019G05140, and Glyma06G20160 as key genes affecting the soybean growth under salt stress.
\end{abstract}

Additional key words: differentially expressed genes, Glycine max, RNA-seq, WGCNA.

\section{Introduction}

Soybean (Glycine max L.) is the most widely planted oil crop in the world, and the global demand for soybean is still increasing (Chaudhary et al. 2015). Soybean contains about $40 \%$ of protein and $21 \%$ of oil, and plays an important role in human diet, animal feed, bio-oil production, and raw material for industrial products (Le et al. 2011). However, various biotic and abiotic stresses affect the soybean growth and development, leading to the decline of soybean yield and quality. Soil salinization affected the development of many crops around the world and its area is still growing (Sharifi et al. 2007). In saline soil, $\mathrm{Na}^{+}$and $\mathrm{Cl}^{-}$are the main causes of toxicity (Munns 2002). $\mathrm{Na}^{+}$is not an essential element for plant growth, and high concentration of $\mathrm{Na}^{+}$ion is toxic to most plants (Tester and Davenport 2003). High concentration of chloride ion can also inhibit the growth of some plants (Geilfus 2018). Soybean is a moderately salt-tolerant crop. Salt stress inhibits the germination and growth of soybean seeds, and hinder the formation of soybean nodules (Wang et al. 1999). When the soil salt content exceeds $5 \mathrm{dS} \mathrm{m}^{-1}$, the yield of soybean would be seriously decreased (Ashraf and Akram 2009). Therefore, research on salt tolerance of soybean is vital for breeding salt-tolerant cultivars.

Transcriptome is the sum of all RNA transcribed by a certain organism under certain environmental conditions. Transcriptome technology plays an important role in explaining gene function, revealing the molecular composition of cells and tissues, and molecular mechanisms of many processes (Luo et al. 2015). Transcriptome sequencing technology can not only accurately determine the expression of known genes, but also detect the sequence and functional classification of unknown genes specific to plants. Several studies have performed transcriptomes sequencing of salt-tolerant plants and screened the salt stress related genes. Long et al. (2015) analyzed the transcriptome of $\mathrm{NaCl}$-treated rape roots and found 163 differentially expressed genes (DEGs), some of which were related to proline metabolism, sugar metabolism, saccharide metabolism, and redox processes. Dang et al. (2013) obtained 65340 DEGs by sequencing the transcriptome of salt-stressed halophyte Reaumuria trigyma, and put these genes into 29 Kyoto encyclopedia of genes and genomes (KEGG) pathways, pointing out that the salt-stress response genes were related to ion transport

Submitted 11 February 2020, last revision 24 June 2020, accepted 25 June 2020.

Abbreviations: DEGs - differentially expressed genes; GO - gene ontology; KEGG - Kyoto encyclopedia of genes and genomes; WGCNA - weighted gene co-expression network analysis.

Acknowledgements: This study was funded by the Agriculture Scientific and Technological Innovation Project of Shandong Academy of Agricultural Sciences (grant No. CXGC2016A02), Agricultural Variety Improvement Project of Shandong Province (grant Nos. 2019LZGC017, 2019LZGC004), and Shandong Province Modern Agricultural Technology System Coarse Cereals Innovation Team. The first two authors equally participate in this work.

*Corresponding author; e-mail: dinghanfengsd@outlook.com 
and reactive oxygen species scavenging. Liu et al. (2018) performed whole transcriptome sequencing on soybean root and leaf, demonstrating that phytohormone signal pathways were triggered and photosynthesis was decreased under salt stress.

In this study, we treated three different soybean cultivars with high and low concentration of $\mathrm{NaCl}$ and performed mRNA sequencing. To identify several potential genes affecting soybean growth under salt stress, we combined the results of transcripts assembly and annotation, weighted gene co-expression network analysis (WGCNA), and DEGs.

\section{Materials and methods}

Plants and treatments: To study the common mechanism of soybean salt tolerance, we used Glycine max (L.) Merr. cultivars: LD (Lindou 10, bred and provided by Linyi Academy of Agricultural Sciences, Shandong, China), QH (Qihuang 34, bred and provided by Crop Research Institute, Shandong Academy of Agricultural Sciences, China), and WD8 (Weidou 8, bred and provided by Weifang Academy of Agricultural Sciences, Shandong, China). Plants were divided into three groups: the control (CK) group, the low concentration (LS) group, and the high concentration (HS) group. Each treatment was repeated three times.

Twenty seven seeds (nine for each cultivar) were individually cultivated in a pot with soil. After the first true leaf unfolding, the seeds were moved into a 1/4 Hoagland nutrient solution and grown under day/night temperatures of $27 / 23{ }^{\circ} \mathrm{C}$, an air humidity of $50 \%$, a light intensity of $30000 \mathrm{~lx}$, and a 17-h photoperiod. After $20 \mathrm{~d}$, the three groups were treated with $1 \mathrm{dm}^{3}$ of $1 / 4$ Hoagland nutrient solution with $0 \mathrm{mM} \mathrm{NaCl}(\mathrm{CK}), 75 \mathrm{mM} \mathrm{NaCl}$ (LS), and $150 \mathrm{mM} \mathrm{NaCl}$ (HS) solution, respectively. Three roots from each treatment were collected as three replicates at the time point of $6 \mathrm{~h}$.

Isolation of RNA, cDNA library preparation, and sequencing: The total RNA was extracted using TRIzol reagent (Thermo Fisher Scientific, Waltham, USA) according to the manufacture's protocol. The quantity of RNA was detected by Nanodrop2000 (Thermo Fisher Scientific) and the purity was detected by agarose gel electrophoresis. The Agilent2100 (Agilent Technologies, Palo Alto, CA, USA) was used to measure the RNA integrity number. The total amount of RNA was $1 \mu \mathrm{g}$ and the absorbance $\mathrm{A}_{260} / \mathrm{A}_{280}$ value ranged from 1.8 to 2.2. The mRNA was isolated from the total RNA using Oligo (dT) magnetic beads paired with poly(A) and randomly cleaved into small fragments by fragmentation buffer (Agilent Technologies). The cDNA library was built with the Truseq ${ }^{\mathrm{TM}}$ RNA sample prep kit (Illumina, San Diego, CA, USA) according to the manufacture's instruction. The sequencing was performed with the HiSeq 2500 system (Illumina).

Analysis of RNA sequencing data and alignment: The SepPrep software (https://github.com/jstjohn/SeqPrep) was used to control the quality of the original reads, and the screening criteria are as follows: 1) removed the adaptor sequence reads and the sequences without fragments insertion due to adaptor self-connection; 2) trimmed the base whose Q-score was $<20$ at the 3 'end of the sequence. The remaining sequence with base whose Q-score was still $<10$ would be also deleted; 3) removed the N-containing reads; 4) discarded the sequences less than $30 \mathrm{bp}$ in length after trimming. The trimmed reads were mapped to the reference genome (NCBI Glycine max_v2.1 GCF_000004515.5) using the HISAT2 software (Kim et al. 2015).

The transcript assembly and annotation: The mapped reads were assembled to a transcriptome using Cufflinks software (http: //cole-trapnell-lab.github.io/cufflinks/), and compared with known transcripts to obtain transcripts without annotation. Functional annotation of potential new transcripts allows us to discover new genes and transcripts, or new exon regions of known genes. Gene ontology $(G O)$ was used to annotate genes and their products according to their participation in biological process (BP), molecular function (MF), and cellular component (CC) to get general understanding of functional classification. Comparing the genes with the Kyoto encyclopedia of genes and genomes $(K E G G)$ database revealed the specific biological pathways that a gene or its transcripts might participate in, and helped to understand the biological functions of genes at the system level.

Differential expression analysis: Read counts of each gene and transcript were obtained with the RSEM 1.3.1 software (Li and Dewey 2011) by using the alignment results and genome annotation files and then performed fragments per kilobase of exon model per million mapped fragments and transcripts per kilobase of exon model per million mapped reads transformation to obtain the standardized gene/transcript expression level. The DESeq2 1.24 package (http://www.bioconductor.org/ packages/DESeq2) in $R$ Bioconductor 3.6 (http://www. bioconductor.org/) was used to analyze the differential expression among different groups. The gene expression of each sample was as input matrix, and the cultivars and salt stress treatments were as phenotypic information. WGCNA 1.67 package (Langfelder and Horvath 2008) was used to discover the modules related to different apparent characetristics. The visualization analysis was performed using Cytoscape 3.7.1 software (Shannon et al. 2003).

Real-time quantitative PCR analysis: The tissue samples were added into the mortar containing liquid nitrogen and ground to fine powder with a pestle. The total RNA was isolated with TRIzol reagent (Invitrogen, Carlsbad, CA, USA). The cDNA was synthesized using reverse transcription kit (Invitrogen), and then amplified using SYBR Green PCR mix (Invitrogen) and the ABI Prism 7300 sequence detection system (Applied Biosystems, Foster City, USA) according to the manufacturer's instructions. The quantitative PCR data were normalized to actin and analyzed with GenEX software (MultiD Analyses $A B$, Goteborg, Sweden). The primer pairs used 
for amplification are shown in Table 1 Suppl.

Data analysis: The statistical analysis of quantitative PCR data was performed by $t$-test with the GraphPad Prism v. 5.0 software for Windows (GraphPad, San Diego, CA, USA). The minimum significance level was set at $\alpha=0.05$.

\section{Results}

The statistical results of sequencing each sample are shown in Table 2 Suppl. Taking the control group of the LD cultivar as an example, the distribution of base error and $\mathrm{A} / \mathrm{T} / \mathrm{C} / \mathrm{G}$ base content are displayed in Fig. 1. The sequencing error rate increased with the length of sequenced reads due to the consumption of reagents in the sequencing process, which is known as common feature of Illumina high-throughput sequencing platform. The $6 \mathrm{bp}$ random primers used in reverse transcription would induce a preference of the first few nucleotides (Fig. 1).

Taking the control group of the LD cultivar (LDCK_1) as an example, we analyzed the sequencing saturation, sequencing coverage, and reads distribution in different genomic regions (Fig. 1). Most of the genes with reads per kilobase per million mapped reads value $>3.5$ were nearly saturated when aligned with $40 \%$ of the sequence reads, indicating that the sequencing could cover most of the expressed genes. Fig. 1 Suppl. showed the coverage of all genes and there was no obvious bias peak, indicating that the sequencing has even distribution. The percentage of reads mapped to genome regions was also shown in Fig. 2 Suppl.
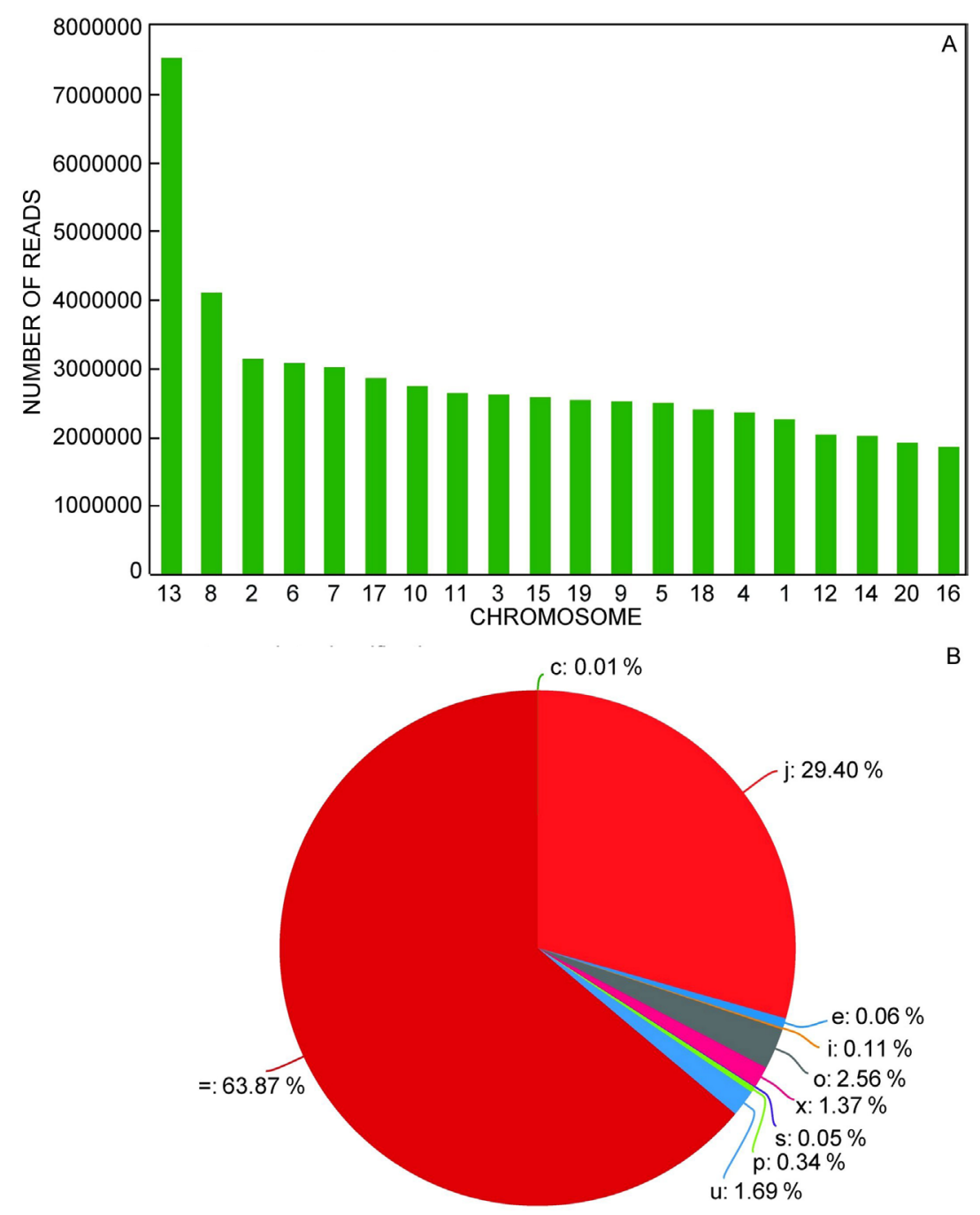

Fig. 1. Assembly of expressed transcripts. $A$ - distribution map of transcription length; $B$ - statistical map of new transcript classification, $=-$ known transcripts consistent with reference annotations, $\mathrm{c}-$ assembled transcripts contained in known transcripts, e - fragments of potential RNA precursors, $\mathrm{i}$ - transcripts fragments completely overlapping introns of reference transcripts, $\mathrm{j}$ - potential new transcripts or transcriptional fragments with at least one junction site consistent with reference transcripts, $o$ - overlapped with the exon of reference transcripts, $\mathrm{p}$ - transcriptional fragments that might be produced by polymerase, $\mathrm{s}$ - introns of transcriptional fragments overlapped with the antisense chain of reference transcript introns, $\mathrm{u}$ - unknown transcripts of intergenic regions, $\mathrm{x}$ - overlapped with the antisense chain of reference transcript exons). 

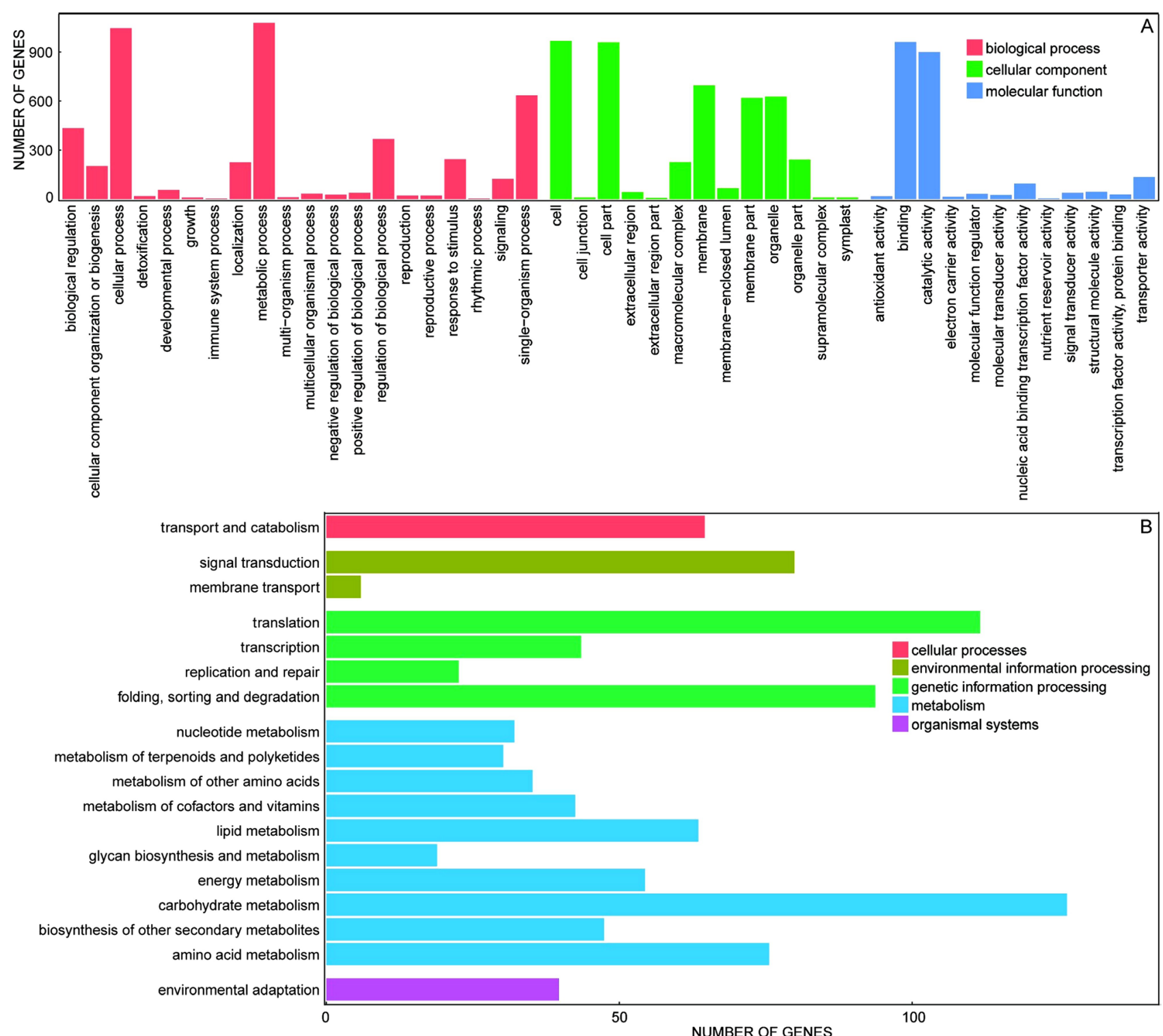

Fig. 2. Classification of the defferentially expressed gene biological functions. $A$ - analysis based on the gene ontology database. $B$ analysis based on the Kyoto encyclopedia of genes and genomes database.

Cufflinks software was used to assemble the results of alignment with the reference genomes, to identify pair end fragments from different spliceosomes, and to search for the overlapping fragments. Transcripts were assembled according to each fragment. The differences between the sequence and the original annotations were obtained by comparing them with the reference transcripts. Fig. $1 A$ showed the distribution of transcript length in chromosomes. Fig. $1 B$ showed the classification statistics of new transcripts. The majority transcripts $(63.87 \%)$ were consistent with reference annotations, and classes of $\mathrm{x}, \mathrm{i}, \mathrm{j}, \mathrm{u}$, and o were defined as new transcripts, while genes in the class of $u$ was defined as new genes.

Then we analyzed the differentially expressed genes (DEGs) between the control group and the high $\mathrm{NaCl}$ concentration group of the three cultivars. 18 804 DEGs were found between CK group and HS group in cv. QH,
18488 DEGs were found between CK group and HS group in cv. WD, and 20172 DEGs were found between CK group and HS group in cv. LD (Fig. 2 Suppl.). In order to show the common genes among the different cultivars, we cross-compared the DEGs and obtained 8383 genes that were commonly expressed in these three cultivars (Fig. 2 Suppl. and https://pan.baidu.com/s/1 zUz0sCTo9GWq6eVEPkn3g, password "0000").

To further understand the potential biological functions of the DEGs and the possible pathways they participate in, we annotated them using $G O$ and $K E G G$ database. According to $G O$ analysis, the transcripts were classified into three groups: 20 in biological processes, 13 in cellular components, and 12 in molecular functions. In biological processes, the two most abundant subcategories were metabolic process and cellular process. For cellular components, cell and cell part were the most dominant 
A

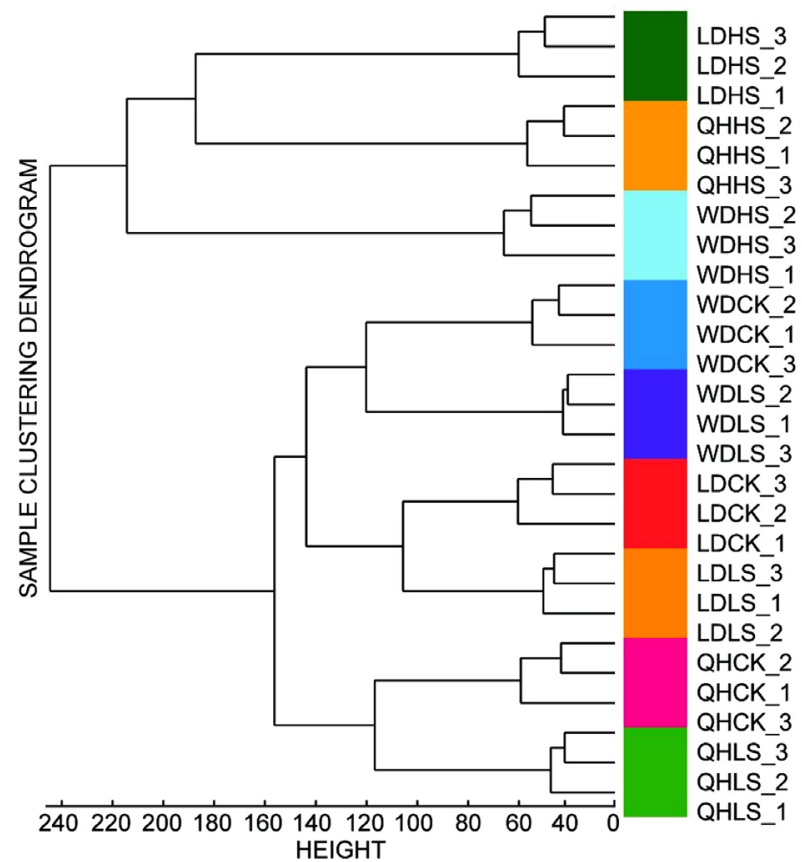

correlation between module and trait

\begin{tabular}{rr} 
MEpurple & 528 \\
MEgreen & 1609 \\
MEtan & 236 \\
MEturquoise & 8878 \\
MEbrown & 3650 \\
MEpink & 1378 \\
MEblack & 1534 \\
\hline MEgreenyellow & 395 \\
MEyellow & 1818 \\
\hline MEmagenta & 758 \\
MEred & 1582 \\
\hline MEblue & 7548 \\
\hline MEsalmon & 209 \\
MEgrey & 726
\end{tabular}

$\begin{array}{llllllllll}-0.182 & 0.121 & -0.53 & -0.272 & -0.197 & 0.499 & -0.166 & 0.272 & 0.454\end{array}$ $0.364)(0.548)(0.00446(0.17)(0.325)(0.00806(0.408)(0.17)(0.0174)$ $\begin{array}{lllllllll}-0.333 & -0.166 & -0.545 & -0.318 & 0 & 0.136 & 0.272 & 0.409 & 0.545\end{array}$ $(0.0896)(0.408)(0.00329(0.106) \quad$ (1) $\quad(0.499)(0.17)(0.0341(0.00329)$ $\begin{array}{lllllllll}0.409 & 0.121 & -0.545 & -0.393 & -0.166 & -0.182 & -0.0605 & 0.363 & 0.454\end{array}$ $0.0341)(0.548)(0.00329(0.0426)(0.408)(0.364)(0.764)(0.0627)(0.0174)$ $\begin{array}{llllllllll}0.106 & 0.136 & 0.469 & -0.151 & -0.545 & 0.469 & -0.348 & -0.212 & 0.287\end{array}$ $\begin{array}{lllllllll}-0.106 & 0.136 & 0.469 & -0.151 & -0.545 & 0.469 & -0.348 & -0.212 & 0.287 \\ -0.079) & (0.499)(0.0136) & (0.452)(0.00329(0.0136)(0.0753) & (0.288) & (0.147) \\ -0.0757 & 0.272 & 0.545 & -0.484 & -0.424 & -0.318 & -0.0454 & 0.121 & 0.409\end{array}$ $\begin{array}{llllllllll}-0.0757 & 0.272 & 0.545 & -0.484 & -0.424 & -0.318 & -0.0454 & 0.121 & 0.409\end{array}$ $0.707)(0.17)(0.00329(0.0105)(0.0275)(0.106)(0.822)(0.548)(0.0341)$ $\begin{array}{lllllllll}-0.242 & 0 & 0.136 & -0.409 & -0.166 & -0.545 & 0.272 & 0.409 & 0.545\end{array}$ $(0.224)$ (1) (0.499) $(0.0341)(0.408)(0.00329(0.17)(0.0341 \times 0.00329) \quad 0.2$

$\begin{array}{lllllllll}-0.212 & 0.0605 & 0.166 & 0.409 & 0.166 & 0.545 & -0.182 & -0.545 & -0.409\end{array}$ 0.288) (0.764) (0.408)(0.0341)(0.408)(0.00329 (0.364)(0.00329(0.0341) $\begin{array}{lllllllll}0.257 & 0.409 & 0.545 & 0.0454 & -0.136 & 0.106 & -0.363 & -0.545 & -0.318\end{array}$ (0.196) $(0.0341$ Y0.00329 (0.822) (0.499) (0.599) $(0.0627$ (0.00329 (0.106) $\begin{array}{llllllllll}0.272 & 0.409 & -0.136 & 0.545 & 0.136 & 0 & -0.272 & -0.409 & -0.545\end{array}$

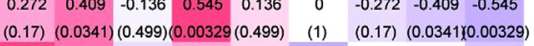
$\begin{array}{lllllllll}0.545 & 0.409 & -0.272 & 0.272 & 0.136 & -0.545 & -0.0454 & -0.0908 & -0.409\end{array}$ $\begin{array}{llll}0.00329(0.0341)(0.17) & (0.17)(0.499)(0.00329(0.822)(0.652)(0.0341)\end{array}$ $\begin{array}{llllllllll}0.197 & -0.0303 & -0.545 & 0.545 & 0.182 & 0.409 & -0.0757 & -0.272 & -0.409\end{array}$ $(0.325)(0.881)(0.003290 .00329(0.364)(0.0341)(0.707)(0.17)(0.0341)$

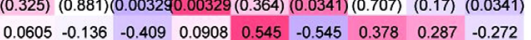
\begin{tabular}{|ccccccc|c|c|}
0.0605 & -0.136 & -0.409 & 0.0908 & 0.545 & -0.545 & 0.378 & 0.287 & -0.272 \\
$(0.764)$ & $(0.499)$ & $(0.0341)(0.652)(0.003290 .00329(0.0519)$ & $(0.147)$ & $(0.17)$
\end{tabular}

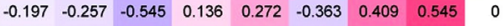
$(0.325)(0.196)(0.00329(0.499)(0.17)(0.0627)(0.0341 \times 0.00329$ (1)

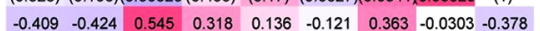

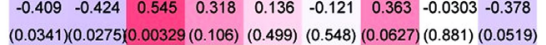

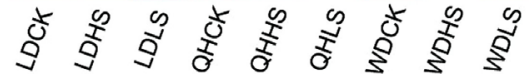

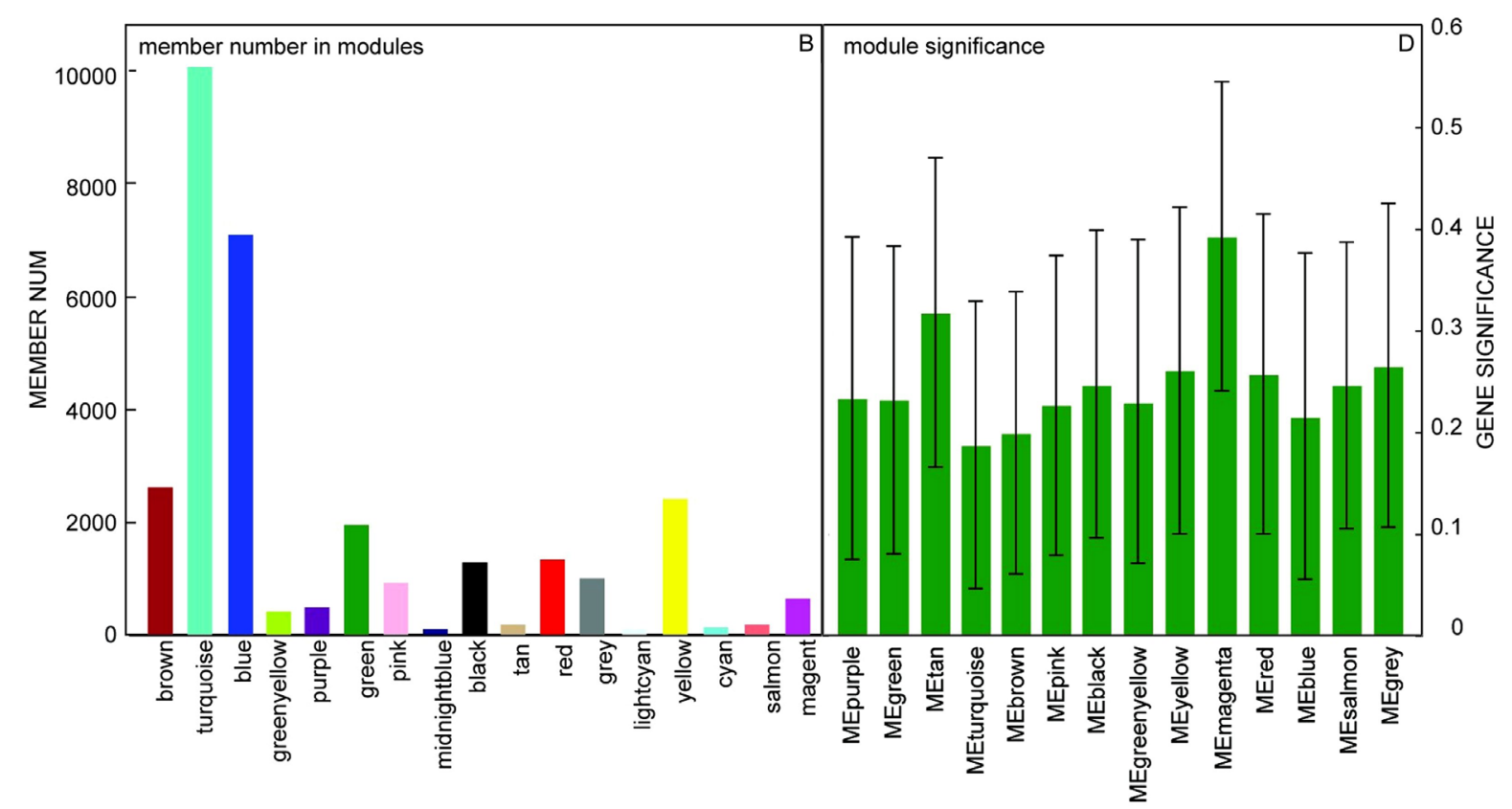

Fig. 3. Weighted gene co-expression network analysis. $A$ - clustering analysis on all samples; $B$ - gene dendrogram and module colors. A height was set to 0.25 . Grey represents genes that could not cluster to any other modules; $C$ - correlation between modules and phenotypes. Red represents a positive correlation, and blue represents a negative correlation. The numbers in the graph are correlation coefficients, and the values in parentheses indicate the significance of correlation coefficients; $D$ - module significance.

subcategories. With regards to molecular function, the dominant terms were binding and catalytic activity (Fig. 2A). As shown in Fig. $2 B$, the DEGs were annotated to five processes based on the $K E G G$ database, including cellular processes, environment information processing, genetic information processing, human diseases, metabolism, and organism systems. And the DEGs were significantly enriched in saccharide metabolism, translation, and folding, sorting, and degradation.
Cluster analysis was performed on all samples. As shown in Fig. $3 A$, there were no outliers, and thus the samples were suitable for further analysis. The expression matrix was transformed into adjacency matrix, then transformed into topological matrix. We used averagelinkage hierarchical clustering method to cluster genes based on transfer occurrence matrix and set the minimum number of bases for each gene network module according to the standard of dynamic tree cut algorithms (Langfelder 


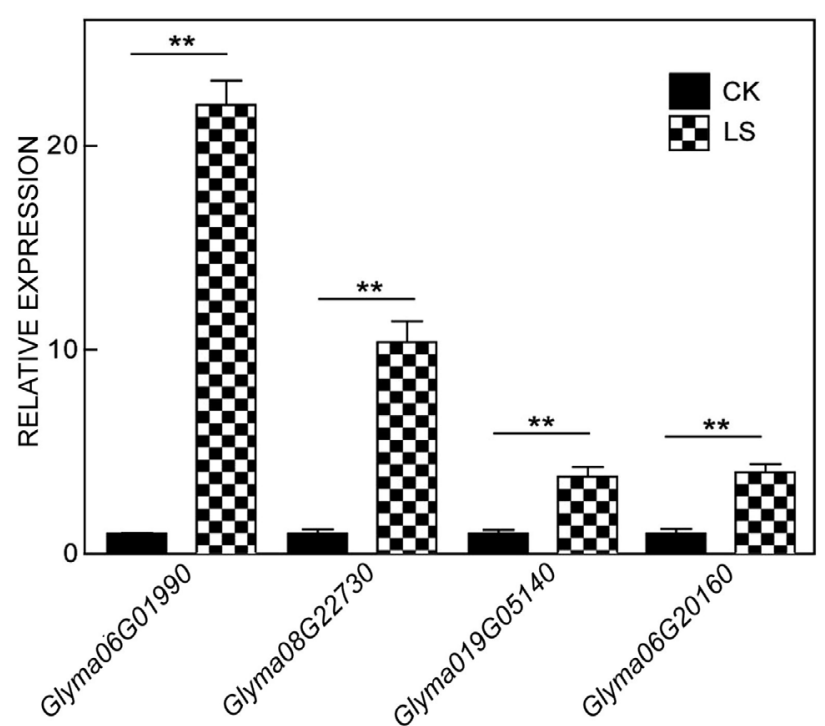

Fig. 4. Verification of gene expression of representative genes by quantitative PCR. Expressions of Glyma06G01990, Glyma08G22730, Glyma019G05140, and Glyma06G20160 of the soybean cultivars treated with $1 \mathrm{dm}^{3}$ of $0 \mathrm{mM} \mathrm{NaCl}(\mathrm{CK})$ and $75 \mathrm{mM} \mathrm{NaCl}$ (LS) for $6 \mathrm{~h}$ were analyzed by quantitative PCR; the gene expressions were normalized to actin. Means \pm SDs, $n=3$, ** - significant differences at $P<0.01$ determined by Student's $t$-test.

et al. 2008). After the gene modules were determined, we calculated the eigengenes of each module in turn and performed clustering analysis, which merged the closer modules into new modules. Seventeen modules were obtained (Fig. 3B). According to the eigenvectors of each module, we calculated the correlation between these modules and the sample phenotypes (Fig. 3C). We also calculated the significance of each module and found the Magenta module in Fig $3 B$ had the most significant correlation with phenotype (Fig. $3 D$ ). To study the gene interaction in this module, we performed visualization analysis (Fig. 3 Suppl.) and screened top 60 genes with strong connection. By cross-comparing these 60 genes with 8383 DEGs of the three cultivars, 35 genes were obtained (Table 3 Suppl.), and we speculated they might relate to the soybean growth under salt stress.

From the 35 genes, 10 of the top differently expressed genes were selected for verification. Among them, the expression of Glyma06G01990, Glyma08G22730, Glyma019G05140, and Glyma06G20160 in LS group were 22.02, 10.38, 3.78, and 4.01 times to that in CK group (Fig. 4). The differences in expression of these genes were consistent with the analysis results mentioned above.

\section{Discussion}

Salinity is one of the most important environmental factors controlling plant growth and development and affecting the crop yield and quality. Plants were damaged by salt stress through osmotic stress, ionic injury and other processes (Yadav et al. 2011). The salt tolerance mechanism of soybean is very complex, belonging to quantitative traits controlled by multiple genes. However, molecular mechanism of salt tolerance in soybean remains not to be completely clear and many key factors have not been discovered yet. It is particularly important to obtain key salt-tolerant genes for bioengineering research. To this purpose, we studied the gene expression in soybean at transcriptome level and analyzed the DEGs under salt stress.

Different organs of the same plant can be selected for salt-tolerant transcriptome sequencing. Most sampling sites are root ( $\mathrm{Gu}$ et al. 2018), leaf (Yuan et al. 2016), and whole seedling (Cui et al. 2018). Root system, absorbing water and mineral nutrition from soil, is the first organ exposed to salt stress thus becomes an ideal material for transcriptome analysis in most plant salt tolerance studies (Yu et al. 2016). Therefore, we also chose root of soybean as the study object in this experiment. The differential gene analysis of soybean root showed there were 8383 overlapping DEGs between control group and high $\mathrm{NaCl}$ concentration group among the three cultivars. The pathway enrichment analysis of these DEGs revealed that saccharide metabolism was significantly enriched. Saccharides, the product of photosynthesis and the substrate for respiration, provides carbon skeleton and energy for plant growth, and ability to enhance stress resistance (Fettke and Fernie 2015). The synthesis and decomposition of saccharides affect osmotic regulation, which relates to the ability of plants to resist stress environment and helps the membrane maintain its integrity and function (Da Silva and Arrabaca 2004). Several studies have investigated the association between salt stress and saccharide metabolism. The activities of acid and neutral invertase, and especially sucrose synthase, were enhanced under salt stress in tomato seedlings of salt-tolerant cultivars (Khelil et al. 2007). Dubey and Singh (1999) found that the activities of sucrose phosphate synthase in salt-sensitive and salttolerant rice cultivars were both enhanced under salt stress. In many plants, salt stress increases the content of soluble sugars such as glucose, sucrose, and starch in leaves, and the negative feedback inhibition of the increasing saccharide content is one of the reasons for the decrease of photosynthesis (Badr et al. 2004). Our findings in this study provided evidence that genes involved in saccharide metabolism had strong association with salt tolerance. Since soluble sugars are signal molecules for plants to adapt to the environment, this may be an adaptation ability of plants to salt tolerance.

According to the WGCNA analysis results, a total of 35 genes were differentially expressed among the three cultivars and most closely related to phenotype. The most significant DEGs (based on the value of $\log 2 \mathrm{fc}$ ), Glyma06G01990, annotated as "glycosyl transferase family 2", was strongly induced under salt stress in this study. Glycosyl transferase plays a vital role in maintaining cell homeostasis. Photoassimilates need to be catalyzed by glycosyl transferases to convert into disaccharides, oligosaccharides and polysaccharides (Lim et al. 2014). Plants produce a large number of secondary metabolites, which are usually modified by glycosylation. Glycosyl transferase participates in plant hypersensitive reaction by 
synthesizing some resistant products such as scopoletin glucoside, scopoletin, and betacyanins under environmental stresses (Lim et al. 2003). Our finding is consistent with the report that glycosyl transferases genes (UGT79B2 and $U G T 79 B 3$ ) were found highly induced by abiotic stresses, including salt, drought and cold stresses (Li et al. 2017). Glyma08G22730, annotated as "desaturase", was up-regulated under salt stress in this study. Hydrophobic lipids, which act as cell barriers, can prevent many ions and macromolecules from entering cells. Salt tolerance of plants depends on their inherent unsaturated fatty acids and their ability to maintain or adjust unsaturated fatty acids, while salt-sensitive plants showed a decrease in oleic acid under salt stress (Im et al. 2002). Maintaining the fluidity of membrane lipids by regulating the activity of desaturase and changing the degree of unsaturation of fatty acids is a characteristic of plant adaptation to stress (Kachroo et al. 2001). Our finding suggested that when soybean is exposed to salt stress, the increasing activity of desaturase might have a role in maintaining the integrity and fluidity of the membrane under salinity. The expression of Glyma03G42140 was induced in response to salt stress. Glyma03G42140 identified in this study had acyl groups transferase activity, which had been reported to be associated with fertility, oil content, and stress resistance in plant (Gidda et al. 2009). Acyltransferase participates in the synthesis of unsaturated fatty acids, which can increase salt tolerance of plants (Gupta et al. 2013). Li et al. (2007) reported that the permeability of young roots and seed coats in Arabidopsis acyltransferase (GPAT5) mutants is highly increased, resulting in water loss under high salinity. Our finding provided evidence that the increased expression of acyltransferases might help enhance salt tolerance when plants were exposed to salt stress. Glyma07G34560, which was annotated as "cytochrome $\mathrm{P}_{450}$ ", was upregulated in this study. Similarly, studies have shown that cytochrome $\mathrm{P}_{450}$ family genes in Populus euphratica and barley were also up-regulated under salt stress (Ueda et al. 2002, Gu et al. 2004). Cytochrome $\mathrm{P}_{450}$ is a multifunctional oxidase widely expressed in plants. It has been reported that selective splicing of genes can be induced by adversity stress, and the expression of gene encoding $\mathrm{P}_{450}$ can be regulated in different splicing forms by a variety of endogenous and exogenous inducers (Shi et al. 2002). Therefore, the salt tolerance of soybean may be improved by using inducers to elevate the expression of cytochrome $\mathrm{P}_{450}$. Glyma19G05140, annotated as "calcium-transporting ATPase", was also highly expressed in response to salt stress. Calcium is not only an essential nutrient element for plants, but also a second messenger that couples extracellular signals with intracellular physiological responses (Wang et al. 2012). Studies have shown that the concentration of $\mathrm{Ca}^{2+}$ in cells fluctuated, resulting in a series of protective physiological reactions thus alleviating the damage by environmental stress (Yang et al. 2010, Wang et al. 2019). Plant anabolism is always weakened and catabolism is strengthened under stress conditions. This is because the amount of required energy for growth and development becomes insufficient, resulting in various physiological processes involving $\mathrm{Ca}^{2+}$ are affected and hydrolysis of macromolecules in plants is enhanced (Gao et al. 2019). Our results combined with these findings suggest that exogenous calcium could be added to salinization soils to restore the balance of saccharide metabolism and alleviate salt damage. Glyma06G20160, which was up-regulated in this study, is annotated with nucleic acid phosphodiester bond hydrolysis activity. The DNA is damaged under various exogenous and endogenous factors such as ionizing radiation, high salt, and cell metabolites, which leads to cell cycle stagnation and apoptosis (Dizdaroglu et al. 2002, Chen et al. 2018). High concentration of salts could induce DNA double strand breaks in Arabidopsis and delayed repair of DNA damage (Cools and De Veylder 2009). In our study, salt stress induced the up-regulation of Glyma06G20160, which might cause DNA damage through its endonuclease activity. Therefore, the future study of plant DNA double strand break repair under various stresses will be helpful to enhance the resistance of soybeans.

In summary, three cultivars of soybean were used in this experiment to study DEGs between growth under optimum conditions and salt stress conditions. Based on the gene annotation and pathways enrichment analysis, six genes were considered to be the critical genes involved in the response to salt stress and the saccharide metabolism was the most affected pathway in soybean. This experiment and other studies on salt tolerance of soybean mainly focused on the seedling stage of soybean. However, it has been reported that the salt tolerance mechanism of soybean at different growth stages differs (Chen et al. 2018), and salt tolerance in bud stage is also the key to ensure the soybean yield in practice. Moreover, a number of salttolerant plants have evolved effective mechanism and corresponding genes in the long-term process of resistance to salt stress, which may be applied to soybean breeding by genetic engineering transformation.

\section{References}

Ashraf, M., Akram, N.A.: Improving salinity tolerance of plants through conventional breeding and genetic engineering: an analytical comparison. - Biotechnol. Adv. 27: 744-752, 2009.

Badr, A.S., Genet, P., Vinit-Dunand, F., Toussaint, M.L., Epron, D., Badot, P.M.:. Effect of copper on growth in cucumber plants (Cucumis sativus) and its relationships with carbohydrate accumulation and changes in ion contents. - Plant Sci. 166: 12-18, 2004.

Chaudhary, J., Patil, G.B., Sonah, H., Deshmukh, R.K., Vuong, T. D., Valliyodan, B., Nguyen, H.T.: Expanding omics resources for improvement of soybean seed composition traits. - Front. Plant Sci. 6: 1021, 2015.

Chen, H.T., Liu, X.Q., Zhang, H.M., Yuan, X.X., Gu, H.P., Cui, X.Y., Chen, X.: Advances in salinity tolerance of soybean: genetic diversity, heredity, and gene identification contribute to improving salinity tolerance. - J. integr. Agr. 17: 83-89, 2018.

Cools, T., De Veylder, L.: DNA stress checkpoint control and plant development. - Curr. Opin. Plant Biol. 12: 23-28, 2009.

Cui, J., Ren, G., Qiao, H., Xiang, X., Huang, L., Chang, J.: Comparative transcriptome analysis of seedling stage of two sorghum cultivars under salt stress. - J. Plant Growth Regul. 
37:1-13, 2018.

Da Silva, J.M., Arrabaca, M.C.: Contributions of soluble carbohydrates to the osmotic adjustment in the $\mathrm{C} 4$ grass Setaria sphacelata: a comparison between rapidly and slowly imposed water stress. - J. Plant Physiol. 161: 551-555, 2004.

Dang, Z.H., Zheng, L.L., Wang, J., Gao, Z., Wu, S.B., Qi, Z., Wang, Y.C.: Transcriptomic profiling of the salt-stress response in the wild recretohalophyte Reaumuria trigyna. BMC Genomics 14: 29, 2013.

Dizdaroglu, M., Jaruga, P., Birincioglu, M., Rodriguez, H.: Free radical-induced damage to DNA: mechanisms and measurement. - Free Radical Biol. Med. 32: 1102-1115, 2002.

Dubey, R.S., Singh, A.K.: Salinity induces accumulation of soluble sugars and alters the activity of sugar metabolising enzymes in rice plants. - Biol. Plant. 42: 233-239, 1999.

Fettke, J., Fernie, A.R.: Intracellular and cell-to-apoplast compartmentation of carbohydrate metabolism. - Trends Plant Sci. 20: 490-497, 2015.

Gao, Q.Y., Xiong, T.T., Li, X.P., Chen, W.X., Zhu, X.Y.: Calcium and calcium sensors in fruit development and ripening. Scientia Hort. 253: 412-421, 2019.

Geilfus, C.M.: Review on the significance of chlorine for crop yield and quality. - Plant Sci. 270: 114-122, 2018.

Gidda, S.K., Shockey, J.M., Rothstein, S.J., Dyer, J.M., Mullen, R.T.: Arabidopsis thaliana GPAT8 and GPAT9 are localized to the ER and possess distinct ER retrieval signals: functional divergence of the dilysine ER retrieval motif in plant cells. Plant Physiol. Biochem. 47: 867-879, 2009.

Gu, C.S., Xu, S., Wang, Z.Q., Liu, L.Q., Zhang, Y.X., Deng, Y.M, Huang, S.Z.: De novo, sequencing, assembly, and analysis of Iris lactea, var. chinensis, roots' transcriptome in response to salt stress. - Plant Physiol. Biochem. 125: 1-12, 2018.

Gu, R.S, Fonseca, S., Puskas, L.G., Hackler, L., Jr., Zvara, A., Dudits, D., Pais, M.S.: Transcript identification and profiling during salt stress and recovery of Populus euphratica. - Tree Physiol. 24: 265-276, 2004.

Gupta, S.M., Pandey, P., Grover, A., Patade, V.Y., Singh, S., Ahmed, Z.: Cloning and characterization of GPAT gene from Lepidium latifolium L.: a step towards translational research in agri-genomics for food and fuel. - Mol. Biol. Rep. 40: 4235-4240, 2013.

Im, Y.J., Han, O., Chung, G.C., Cho, B.H.: Antisense expression of an Arabidopsis $\omega$-3 fatty acid desaturase gene reduces salt/ drought tolerance in transgenic tobacco plants. - Mol. Cells 13: 264-271, 2002.

Kachroo, P., Shanklin, J., Shah, J., Whittle, E.J., Klessig, D.F.: A fatty acid desaturase modulates the activation of defense signaling pathways in plants. - Proc. nat. Acad. Sci. USA 98: 9448-9453, 2001.

Khelil, A., Menu, T., Ricard, B.: Adaptive response to salt involving carbohydrate metabolism in leaves of a saltsensitive tomato cultivar. - Plant Physiol. Biochem. 45: 551$559,2007$.

Kim, D., Langmead, B., Salzberg, S.L.: HISAT: a fast spliced aligner with low memory requirements. - Nat. Methods 12: 357-360, 2015.

Langfelder, P., Horvath, S.: WGCNA: an R package for weighted correlation network analysis. - BMC Bioinformatics 9: 559, 2008.

Langfelder, P., Zhang, B., Horvath, S.: Defining clusters from a hierarchical cluster tree: the dynamic tree cut package for R. Bioinformatics 24: 719-720, 2008.

Le, D.T., Nishiyama, R., Watanabe, Y., Mochida, K., YamaguchiShinozaki, K., Shinozaki, K., Tran, L.S.: Genome-wide survey and expression analysis of the plant-specific NAC transcription factor family in soybean during development and dehydration stress. - DNA Res. 18: 263-276, 2011.

Li, B., Dewey, C.N.: RSEM: accurate transcript quantification from RNA-Seq data with or without a reference genome. BMC Bioinformatics 12: 323, 2011.

Li, P., Li, Y.J., Zhang, F.J., Zhang, G.Z., Jiang, X.Y., Yu, H.M., Hou, B.K.: The Arabidopsis UDP-glycosyltransferases UGT79B2 and UGT79B3, contribute to cold, salt and drought stress tolerance via modulating anthocyanin accumulation. Plant J. 89: 85-103, 2017.

Li, Y.H., Beisson, F., Koo, A.J., Molina, I., Pollard, M., Ohlrogge, J.: Identification of acyltransferases required for cutin biosynthesis and production of cutin with suberin-like monomers. - Proc. nat. Acad. Sci. USA 104: 18339-18344, 2007.

Lim, E.K., Baldauf, S., Li, Y., Elias, L., Worrall, D., Spencer, S.P., Jackson, R.G., Taguchi, G., Ross, J., Bowles, D.J.: Evolution of substrate recognition across a multigene family of glycosyltransferases in Arabidopsis. - Glycobiology 13: 139-145, 2003.

Lim, E.K., Bowles, D.J.: A class of plant glycosyltransferases involved in cellular homeostasis. - Embo J. 23: 2915-2922, 2014.

Liu, A.L., Xiao, Z.X., Li, M.W., Wong, F.L., Yung, W.S., Ku, Y.S., Wang, Q.W., Wang, X., Xie, M., Yim, A.K., Chan, T.F., Lam, H.M.: Transcriptomic reprogramming in soybean seedlings under salt stress. - Plant Cell Environ. 42: 98-114, 2018.

Long, W.H., Zou, X.L., Zhang, X.K.: Transcriptome analysis of canola (Brassica napus) under salt stress at the germination stage. - PloS ONE 10: e0116217, 2015.

Luo, Y.P., Coskun, V., Liang, A.B., Yu, J.H., Cheng, L.M., Ge, W.H., Shi, Z.P., Zhang, K.S., Li, C., Cui, Y., Lin, H.J., Luo, D.D., Wang, J.B., Lin, C., Dai, Z., Zhu, H.W., Zhang, J., Liu, J., Liu, H.L., De Vellis, J., Horvath, S., Sun, Y.E., Li, S.G.: Single-cell transcriptome analyses reveal signals to activate dormant neural stem cells. - Cell 161: 1175-1186, 2015.

Munns, R.: Comparative physiology of salt and water stress. Plant Cell Environ. 25: 239-250, 2002.

Shannon, P., Markiel, A., Ozier, O., Baliga, N.S., Wang, J.T., Ramage, D., Amin, N., Schwikowski, B., Ideker, T.: Cytoscape: a software environment for integrated models of biomolecular interaction networks. - Genome Res. 13: 24982504, 2003.

Sharifi, M., Ghorbanli, M., Ebrahimzadeh, H.: Improved growth of salinity-stressed soybean after inoculation with salt pretreated mycorrhizal fungi. - J. Plant Physiol. 164: 1144-1151, 2007.

Shi, H.Z., Xiong, L.M., Stevenson, B., Lu, T.G., Zhu, J.K.: The Arabidopsis salt overly sensitive 4 mutants uncover a critical role for vitamin B6 in plant salt tolerance. - Plant Cell 14: 575-588, 2002.

Tester, M., Davenport, R.: $\mathrm{Na}^{+}$tolerance and $\mathrm{Na}^{+}$transport in higher plants. - Ann. Bot. 91: 503-527, 2003.

Ueda, A., Shi, W.M., Nakamura, T., Takabe, T.: Analysis of saltinducible genes in barley roots by differential display. - J. Plant Res. 115: 119-130, 2002.

Wang, D., Shannon, M.C.: Emergence and seedling growth of soybean cultivars and maturity groups under salinity. - Plant Soil 214: 117-124, 1999.

Wang, F., Wan, S.B., Meng, Q.W., Li, X.G.: Regulation of $\mathrm{Ca}^{2+}$ in plant response mechanisms under salt stress. - Life Sci. Res. 16: 362-367, 2012.

Wang, X.P., Zhu, B.P., Jiang, Z.G., Wang, S.C.: Calciummediation of jasmonate biosynthesis and signaling in plants. - Plant Sci. 287: 110192, 2019.

Yadav, S., Irfan, M., Ahmad, A., Hayat, S.: Causes of salinity and 
plant manifestations to salt stress: a review. - J environ. Biol. 32: 667-685, 2011

Yang, F.J., Li, T.L., Zang, Z.J., Lu, S.W.: Effects of timing of exogenous calcium application on the alleviation of salt stress in the tomato seedlings. - Scientia agr. sin. 43: 1181-1188, 2010.

Yu, C.G., Xu, S., Yin, Y.L.: Transcriptome analysis of the
Taxodium 'Zhongshanshan 405 ' roots in response to salinity stress. - Plant Physiol. Biochem. 100: 156-165, 2016.

Yuan, F., Lyu, M.J., Leng, B.Y., Zhu, X.G., Wang, B.S.: The transcriptome of NaCl-treated Limonium bicolor leaves reveals the genes controlling salt secretion of salt gland. Plant mol. Biol. 91: 241-256, 2016. 